\title{
Effect of Polyoxyethylene-Modified Superoxide Dismutase on Recovery of Myocardial Dysfunction after Coronary Stenosis in Dogs
}

\author{
Katsuhiko NOGUCHI, Yoshihiko OJIRI ${ }^{1}$, Noriko KINJO², \\ Hidehiko MOROMIZATO3${ }^{3}$, Junko NAKASONE and Matao SAKANASHI \\ Department of Pharmacology. School of Medicine. \\ 'Laboratory of Physiology and Pharmacology. School of Health Sciences. \\ Departments of ${ }^{2}$ Pediatrics and ${ }^{3}$ Obstetrics and Gynecology. School of Medicine, \\ Faculty of Medicine, University of the Ryukyus, Okinawa 903-01, Japan
}

Accepted July 17, 1990

\begin{abstract}
The effects of superoxide dismutase modified with polyoxyethylene (SOD-POE) on impairment of myocardial segment shortening (SS) during coronary stenosis plus pacing-induced tachycardia (CSPT) and the recovery after reperfusion was examined in anesthetized dogs. SOD-POE or saline was administered i.v. 30 min before reperfusion. Changes in hemodynamic variables and SS by CSPT were similar in both groups. However. the SOD-POE group showed improved recovery of SS compared with the control group. Results indicate that oxygenderived free radicals may partially be involved in the genesis of myocardial dysfunction after CSPT-induced regional ischemia.
\end{abstract}

Prolonged regional myocardial dysfunction occurring after brief coronary artery occlusion has been termed "myocardial stunning". Recently, such reversible contractile dysfunction has also been observed during recovery from exercise-induced regional ischemia in dogs with coronary stenosis $(1,2)$. Although the mechanism responsible for this phenomenon remains unclear, several studies have reported enhancement of the recovery of the stunned myocardium with treatment of oxygen free radical scavengers, which are represented as superoxide dismutase (SOD). and have suggested that oxygen-derived free radicals, such as superoxide anion and hydroxyl radical. play an important role in mediating myocardial injury during coronary occlusion and reperfusion (3-6). However, all these studies in which the effects of the scavengers were examined have been performed in models with a total coronary occlusion followed by reperfusion, and thus these agents might be difficult to access the ischemic myocardium during coronary occlusion. Additionally, relatively high doses of SOD were required to show an appreciable effect in these previous experiments, because of its short plasma half life. According to recent reports, cross-linkage of SOD with activated polyoxyethylene (POE) elongates its circulatory half-life to 7 or $11 \mathrm{hr}$ from the 5-10 min of the unmodified $\operatorname{SOD}(7,8)$, and protective effects of this modified SOD (SOD-POE) on reperfusion induced arrhythmias have been demonstrated (7,9). In the present study. we examined whether the modified SOD enhances recovery of regional myocardial dysfunction produced by coronary stenosis plus pacing stress in anesthetized open-chest dogs.

Mongrel dogs of either sex weighing 10$18 \mathrm{~kg}$ were anesthetized with pentobarbital sodium at $25 \mathrm{mg} / \mathrm{kg}$, i.v. and then ventilated by a respirator (Harvard, model 607). The experimental preparation was similar to that used in our previous study (10). Briefly, a left thoracotomy was performed through the fifth intercostal space. The left circumflex coronary artery (LCX) was dissected free near its origin, and a snare-type occluder was placed around it. An electromagnetic flow probe (Statham, SP7515) was placed 
proximal to the occluder and connected to an electromagnetic flowmeter (Statham, SP2204) for measurement of LCX flow. Aortic and left ventricular pressure were recorded by using a pressure transducer (Statham. P23ID) and a catheter-tip manometer (Millar, PC-350), respectively. Two pairs of piezoelectric crystals $(5 \mathrm{MHz})$ were implanted in the subendocardial regions of the myocardium perfused by the left anterior descending coronary artery (LAD) and the LCX to measure myocardial segment shortening in a circumferential plane, respectively. The leads of each crystal were connected to an ultrasonic amplifier (Mecc, UDM-5B). Percentage segment shortening (SS), an index of regional myocardial function. was calculated from the following equation: $S S=$ $100 \times(E D L-E S L) / E D L$, where EDL and ESL are end-diastolic and end-systolic segment lengths, respectively. In 14 dogs, catheters were inserted into the aorta and the coronary sinus for withdrawal of arterial and coronary sinus blood samples, respectively. The plasma lactate concentration was measured by enzymatic analysis (Lactate Test BMY, Boehringer Mannheim). SOD activities in arterial plasma samples were determined in 4 dogs of the SOD-POE group and 3 dogs of the saline group according to the method of McCord and Fridovich (11). SOD-POE was obtained from Ajinomoto Co., Inc. (Kawasaki, Japan).

Control hemodynamics and regional myocardial shortening were recorded following a stabilization period of at least $30 \mathrm{~min}$. The LCX was then gradually constricted with the occluder to reduce resting LCX flow, and stenosis of the LCX was made sufficient to decrease $S S$ in the region perfused by the LCX to around 1-3\%. After establishing an appropriate degree of hypofunction in the LCX-perfused area, the hemodynamic variables and regional myocardial function were recorded. Then SOD-POE $(1,000 \mathrm{U} / \mathrm{kg}, \mathrm{n}=9)$ or physiological saline $(0.9 \% \mathrm{NaCl} ; 0.5 \mathrm{ml})$ $\mathrm{kg}, \mathrm{n}=9$ ) was administered intravenously. The average time between the beginning of coronary stenosis and the administration in the SOD-POE group and saline group was $32.8 \pm 3.4 \mathrm{~min}$ and $33.0 \pm 3.4 \mathrm{~min}$, respectively. Drug or saline administration was followed after $15 \mathrm{~min}$ by left atrial pacing for $15 \mathrm{~min}$ in order to increase the heart rate by $25 \%$ of the resting rate just before pacing. The coronary stenosis was released immediately after the end of the pacing period. Recordings were obtained until $3 \mathrm{hr}$ after the release of the stenosis.

Time sequence data were analyzed by twoway analysis of variance. The unpaired Student's $t$-test was used to compare values

Table 1. Baseline values of hemodynamic and functional variables

\begin{tabular}{lcc} 
& Saline group $(\mathrm{n}=9)$ & SOD-POE group $(n=9)$ \\
\hline HR (beats/min) & $152 \pm 6$ & $158 \pm 10$ \\
MAOP $(\mathrm{mmHg})$ & $117 \pm 6$ & $112 \pm 2$ \\
MCBF $(\mathrm{ml} / \mathrm{min})$ & $17.4 \pm 1.6$ & $22.3 \pm 3.0$ \\
LVEDP $(\mathrm{mmHg})$ & $5.4 \pm 0.5$ & $4.5 \pm 0.4$ \\
$(+)$ LVdP $/ \mathrm{dt}(\mathrm{mmHg} / \mathrm{sec})$ & $2242 \pm 150$ & $2390 \pm 223$ \\
RP product $(\times 1,000)$ & $20.8 \pm 1.7$ & $20.5 \pm 1.5$ \\
LAD-EDL $(\mathrm{mm})$ & $9.5 \pm 0.6$ & $9.5 \pm 0.7$ \\
LAD-SS $(\%)$ & $17.2 \pm 1.4$ & $17.2 \pm 1.7$ \\
LCX-EDL $(\mathrm{mm})$ & $9.6 \pm 0.8$ & $10.6 \pm 0.5$ \\
LCX-SS $(\%)$ & $14.0 \pm 1.4$ & $12.2 \pm 1.2$ \\
\hline
\end{tabular}

$H R=$ heart rate, $M A O P=$ mean aortic pressure, $M C B F=$ mean blood flow of the left circumflex coronary artery, LVEDP=left ventricular end-diastolic pressure. $(+) \mathrm{LVdP} / \mathrm{dt}=$ peak positive left ventricular $\mathrm{dP} / \mathrm{dt}$. RP product $=$ the product of heart rate and svstolic aortic pressure. LAD-EDL=end-diastolic length in the left anterior descending coronary artery-perfused region. LAD-SS=percent segment shortening in the $L A D$-perfused region, LCX-EDL=end-diastolic length in the left circumflex coronary artery-perfused region, $L C X-S S=$ percent segment shortening in LCX-perfused region. The data are expressed as means \pm S.E. 
between saline and drug administered dogs. All data are expressed as means \pm S.E.

Baseline values of all measured parameters were not significantly different between the saline and SOD-POE groups (Table 1). As shown in Fig. 1, partial occlusion of the LCX reduced the resting flow of the $L C X$ in the saline and SOD-POE groups to $59 \pm 4 \%$ and $50 \pm 6 \%$, respectively, and markedly impaired regional myocardial function in the area distal to the stenosis. Administration of SODPOE as well as saline did not cause any

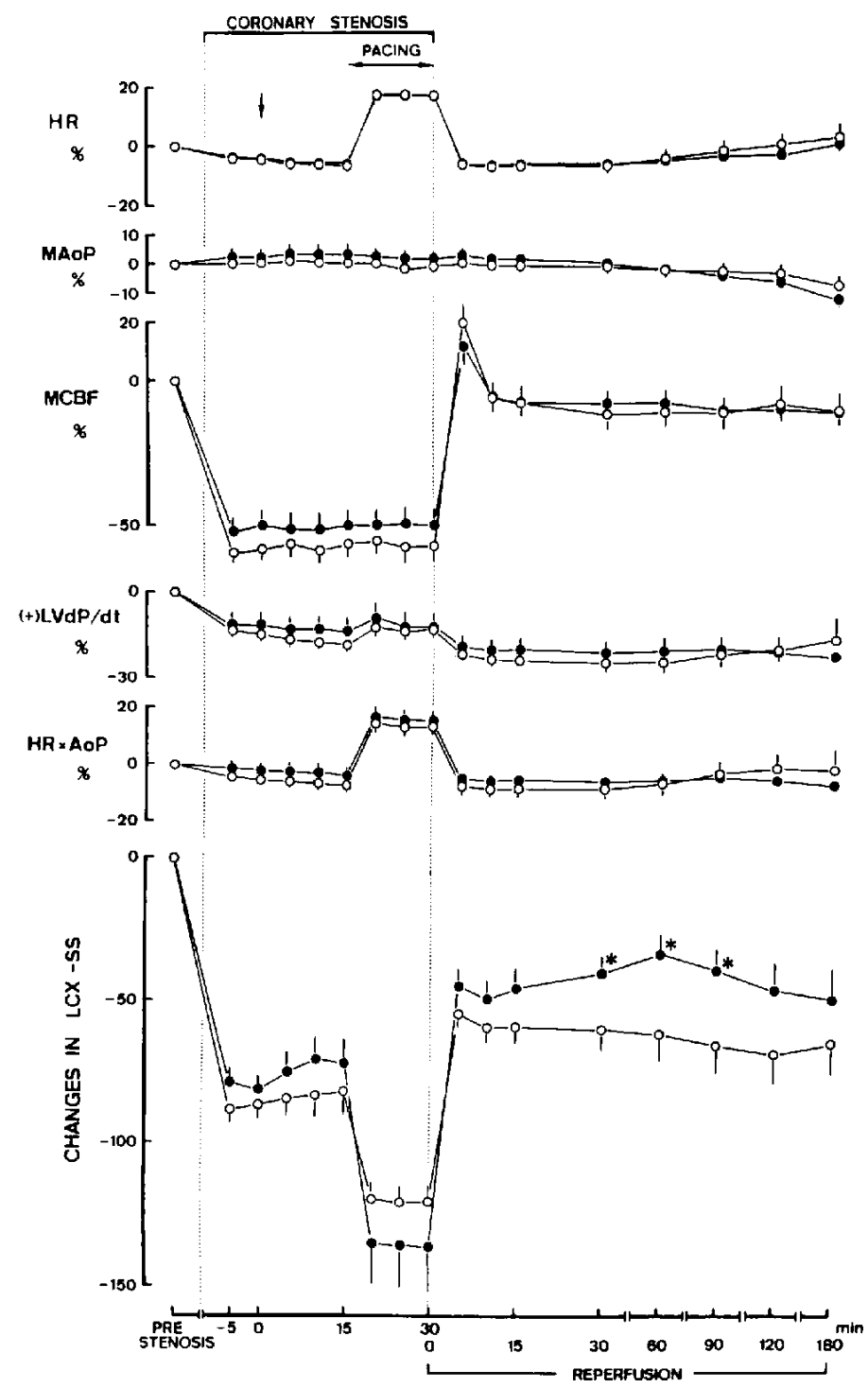

Fig. 1. Percent changes in hemodynamics and myocardial shortening from the prestenosis value. $\mathrm{HR}=$ heart rate, $\mathrm{MAOP}=$ mean aortic pressure, $\mathrm{MCBF}=$ mean blood flow of the left circumflex coronary artery $(L C X),(\div) L V d P / d t=$ peak positive left ventricular $d P / d t . H R \times A O P=$ the product of heart rate and systolic aortic pressure, LCX-SS-segment shortening in LCX-perfused region. The arrow indicates the injection of SOD-POE (1.000 U/kg, i.v., closed circles, $n=9)$ or saline (open circles, $n=9$ ). $\quad *<0.05$, when compared with the data during reperfusion between the SOD-POE and saline groups by two-way analysis of variance. The data are expressed as means \pm S.E. 
appreciable change during coronary stenosis in all measured parameters. Increasing heart rate by atrial pacing for $15 \mathrm{~min}$ in the presence of coronary artery stenosis resulted in a further decrease of SS in the LCX-perfused region and consequently in showing systolic bulging in almost dogs (Fig. 1). Changes in all hemodynamic variables and segment shortenings produced by coronary stenosis plus additional cardiac pacing were similar in both groups (Fig. 1). Following release of the stenosis immediately after the end of the pacing period, heart rate, LCX flow and double products returned to nearly the prestenosis levels in both groups. Although full recovery of segment shortening in the LCX-perfused myocardium was not seen until $3 \mathrm{hr}$ after reperfusion. SOD-POE treated dogs demonstrated significantly improved recovery of this parameter when compared with the saline group at 0.5 .1 and $1.5 \mathrm{hr}$ of reperfusion (Fig. 1). On the other hand, changes in SS in LAD-perfused (nonischemic) myocardium during coronary stenosis and after the release of stenosis were similar in these two groups. There was no significant difference in cardiac lactate extraction between the two groups at any time throughout the experiments.

Plasma SOD activities in dogs receiving SOD-POE $(n=4)$ at $15 \mathrm{~min}, 35 \mathrm{~min}(5 \mathrm{~min}$ after reperfusion), $90 \mathrm{~min} \mathrm{(1} \mathrm{hr}$ after reperfusion) and $210 \mathrm{~min}(3 \mathrm{hr}$ after reperfusion) after the administration apparently increased $(P<0.01)$ from $5.9 \pm 1.8 \mathrm{U} / \mathrm{ml}$ of the control value to $26.3 \pm 3.6 \mathrm{U} / \mathrm{ml}, 19.3 \pm 3.2$ $\mathrm{U} / \mathrm{ml}, 18.5 \pm 3.9 \mathrm{U} / \mathrm{ml}$ and $15.2 \pm 4.9 \mathrm{U} / \mathrm{ml}$. respectively, while those in the saline administered dogs $(n=3)$ remained unchanged, being near the control value $(1.8 \pm 0.6 \mathrm{U} / \mathrm{ml})$.

We found that the long-acting SOD given in a bolus $30 \mathrm{~min}$ before release of coronary stenosis significantly improved contractile dysfunction during the recovery period. There were no significant differences in both hemodynamic variables and cardiac lactate metabolism between the SOD-POE and the saline groups, whereas apparent increases in plasma SOD activities were observed only in the SOD-POE group. Thus, it is likely that the improved recovery of dysfunction seen in dogs with SOD-POE may be attributable at least partly to scavenging oxygen-derived free radicals which would include not only superoxide anion presumably but also more cytotoxic hydroxyl radical generated through the superoxide-driven Fenton reaction. These results are in agreement with recent studies $(3-5,12)$ that scavengers of oxygen-derived free radicals exerted beneficial effects on postischemic dysfunction following reversible myocardial ischemia, implying that the accumulation of reactive oxygen metabolites may be involved in the pathogenesis of myocardial stunning. Furthermore, the SODPOE inhibitable myocardial dysfunction seen in this study may relate to ischemia-induced endothelial damage and/or accumulation of activated leukocytes that would cause generation of these radicals or capillary plugging (13), since SOD-POE probably acts only in the vascular space. However, it should be noted that the data obtained with the experimental model employed in the present study may show some differences from the previous investigations, demonstrating the beneficial action of radical scavenging agents on persistent myocardial dysfunction following ischemia produced by a total coronary artery occlusion and reperfusion. The damage of the ischemic myocardium resulting from coronary stenosis plus pacing stress in the present study appears to be less severe than those in the previous ones (3-5), because these studies have shown that regional functions during the recovery period after a $15 \mathrm{~min}$ coronary occlusion exhibit near akinesis or even systolic bulging (or thinning), whereas those seen in this study manifested only hypofunction (Fig. 1). Relatively moderate effects of the agent reported here as compared to those in these previous studies may be attributable to differences in the experimental preparation and protocol described above. Additionally, the dose of the compound might be insufficient to cause the maximal effect, although the dose employed in this study was determined by taking into account the results of the previous experiment (7) demonstrating that SOD-POE $(1,000 \mathrm{U} / \mathrm{kg}$. i.v.) but not the native SOD $(1,000 \mathrm{U} / \mathrm{kg}$, i.v.) prevented the occurrence of reperfusion arrhythmias in anesthetized rats. 
In the present study, it remains undetermined when the critical free-radical mediated myocardial injury took place, during ischemia, after reperfusion, or both, because SOD-POE has a long lasting activity. Gross et al. (5) have suggested that part of the effect of the scavengers may occur during the ischemic period. In fact, it has been demonstrated in canine models of myocardial ischemia that oxygen free radicals are generated not only after but also during ischemia (14, 15). On the contrary, no significant effect of SOD was observed during the ischemic period in this study, as seen in others $(3,4)$. Recently. Bolli et al. (12) have demonstrated in vivo, by the use of electron magnetic resonance spectroscopy and the spin trap. that a substantial portion of the damage responsible for myocardial stunning develops in the initial seconds of reperfusion following total coronary occlusion. These findings therefore suggest that scavenging of the reactive oxygen free radicals during the ischemic period is not a major factor in protecting against the genesis of postischemic contractile dysfunction. However, it is unknown whether this is the case for the myocardial ischemia produced by increased myocardial axygen consumption under a limited blood supply due to partial coronary occlusion. which is an analogous circumstance to that seen in this experimental model.

Acknowledgment: This study was supported in part by a Grant-in-Aid for Scientific Research (No. 01480502) from the Ministry of Education. Science. and Culture, Japan.

\section{References}

1 Homans, D.C., Sublett, E., Dai, X.-Z. and Bache, R.J.: Persistence of regional left ventricular dysfunction after exercise-induced myocardial ischemia. J. Clin. Invest. 77, 66-73 (1986)

2 Thaulow, E., Guth, B.D., Heusch, G., Gilpin, E., Schulz, R., Kroeger, K. and Ross, J., Jr.: Characteristics of regional myocardial stunning after exercise in dogs with chronic coronary stenosis. Am. J. Physiol. 257, H113-H119 (1989)

3 Myers, M.L., Bolli, R., Lekich, R.F., Hartley, C.J. and Roberts, R.: Enhancement of recovery of myocardial function by oxygen free-radical scavengers after reversible regional ischemia. Circulation 72, 915-921 (1985)

4 Przyklenk, K. and Kloner, R.A.: Superoxide dismutase plus catalase improve contractile function in the canine model of the "stunned myocardium". Circ. Res. 58, 148-156 (1986)

5 Gross, G.J., Farber, N.E., Hardman, H.F. and Warltier, D.C.: Beneficial actions of superoxide dismutase and catalase in stunned myocardium of dogs. Am. J. Physiol. 250, H372-H377 (1986)

6 Bolli, R., Zhu, W.-X., Hartley, C.J., Michael, L.H., Repine, J.E., Hess, M.L., Kukreja, R.C. and Roberts, R.: Attenuation of dysfunction in the postischemic 'stunned' myocardium by dimethylthiourea. Circulation 76, 458-468 (1987)

7 Galcia-Alves, M., Kadowaki, Y., Iwashita, Y. and Nishi, K.: Pretreatment with a single bolus injection of polyoxyethylene-modified superoxide dismutase prevents reperfusion induced arrhythmias in the anesthetized rat. Japan. J. Pharmacol. 51, 199-209 (1989)

8 Morimoto, H., Tsuji, T., Yokoyama, M. and Iwashita, Y.: Preparation of polyoxyethylenemodified superoxide dismutase from single component superoxide dismutase. In Proceedings of the 4th Biennial General Meeting of the Society for Free Radical Research, Edited by Hayashi, O.. Niki, E., Kondo, M. and Yoshikawa, T., p. 615-618. Elsevier. Amsterdam (1989)

9 Yamakawa, T., Kadowaki, Y., Garcia-Alves, M. Yokoyama, M., Iwashita, Y. and Nishi, K.: Effects of polyoxyethylene-modified superoxide dismutase on reperfusion induced arrhythmias in isolated rat and guinea-pig hearts. J. Mol. Cell. Cardiol. 21, 441-452 (1989)

10 Noguchi, K. and Sakanashi, M.: Effects of nipradilol on myocardial ischaemia produced by coronary stenosis in dogs. Br. J. Pharmacol. 91, 411-419 (1987)

11 McCord, J.M. and Fridovich, I.: Superoxide dismutase: An enzymic function for erythrocuprein (hemocuprein). J. Biol. Chem. 244, 6049 6055 (1969)

12 Bolli, R., Jeroudi, M.O., Patel, B.S., Aruoma, O.I, Halliwell, B., Lai, E.K. and McCay, P.B.: Marked reduction of free radical generation and contractile dysfunction by antioxidant therapy begun at the time of reperfusion. Evidence that myocardial "stunning" is a manifestation of reperfusion injury. Circ. Res. 65, 607-622 (1989)

13 Werns, S.W., Shea, M.J. and Lucchesi, B.R.: Free radicals and myocardial injury: pharmacologic implications. Circulation 74, 1-5 (1986)

14 Rao, P.S., Cohen, M.V. and Mueller, H.S.: Production of free radicals and lipid peroxides in early experimental myocardial ischemia. J. Mol. Cell. Cardiol. 16, 713-716 (1983) 
15 Bolli, R., Patel, B.S., Jeroudi, M.O., Lai, E.K. and McCay, P.B.: Demonstration of free radical generation in "stunned" myocardium of intact dogs with the use of the spin trap $\alpha$-phenyl $\mathrm{N}$ tert-butyl nitrone. J. Clin. Invest. 82, 476-485 (1988) 